PROCEEDINGS OF THE

AMERICAN MATHEMATICAL SOCIETY

Volume 131, Number 10, Pages 3009-3017

S 0002-9939(03)07038-2

Article electronically published on March 11, 2003

\title{
CONVERGENCE OF SEQUENCES OF SETS OF ASSOCIATED PRIMES
}

\author{
RODNEY Y. SHARP
}

(Communicated by Bernd Ulrich)

\begin{abstract}
It is a well-known result of M. Brodmann that if $\mathfrak{a}$ is an ideal of a commutative Noetherian ring $A$, then the set of associated primes $\operatorname{Ass}\left(A / \mathfrak{a}^{n}\right)$ of the $n$-th power of $\mathfrak{a}$ is constant for all large $n$. This paper is concerned with the following question: given a prime ideal $\mathfrak{p}$ of $A$ which is known to be in $\operatorname{Ass}\left(A / \mathfrak{a}^{n}\right)$ for all large integers $n$, can one identify a term of the sequence $\left(\operatorname{Ass}\left(A / \mathfrak{a}^{n}\right)\right)_{n \in \mathbb{N}}$ beyond which $\mathfrak{p}$ will subsequently be an ever-present? This paper presents some results about convergence of sequences of sets of associated primes of graded components of finitely generated graded modules over a standard positively graded commutative Noetherian ring; those results are then applied to the above question.
\end{abstract}

\section{INTRODUCTION}

Let $\mathfrak{a}$ be an ideal of a commutative Noetherian ring $A$. It is a well-known result of M. Brodmann [1] that the sequences $\left(\operatorname{Ass}\left(A / \mathfrak{a}^{n}\right)\right)_{n \in \mathbb{N}}$ and $\left(\operatorname{Ass}\left(\mathfrak{a}^{n} / \mathfrak{a}^{n+1}\right)\right)_{n \in \mathbb{N}_{0}}$ have ultimate constant values, denoted here by $\operatorname{Ass}^{*}(\mathfrak{a})$ and $\operatorname{Bss}^{*}(\mathfrak{a})$, respectively. (We use $\mathbb{N}, \mathbb{N}_{0}$ and $\mathbb{Z}$ to denote the sets of positive, non-negative and all integers, respectively.) This paper is about the way in which these sequences converge.

Neither sequence need be monotonic; see S. McAdam and P. Eakin [4, p. 80]. In that paper, McAdam and Eakin obtained a great deal of positive information about the convergence of the two sequences, some of which can be phrased in terms of the (ordinary) Rees ring $\mathcal{R}(\mathfrak{a})$ or the associated graded ring $\mathcal{G}(\mathfrak{a})$ of $\mathfrak{a}$. Let $\mathcal{R}(\mathfrak{a})_{+}$ denote the irrelevant ideal of $\mathcal{R}(\mathfrak{a})$; recall that $\operatorname{Proj}(\mathcal{R}(\mathfrak{a}))$ denotes the set of graded prime ideals of $\mathcal{R}(\mathfrak{a})$ which do not contain the irrelevant ideal. Also, for a graded $\mathcal{R}(\mathfrak{a})$-module $H$, let $a_{\mathcal{R}(\mathfrak{a})_{+}}^{0}(H)$ denote the supremum of the degrees of non-zero homogeneous elements of the graded submodule

$$
\Gamma_{\mathcal{R}(\mathfrak{a})_{+}}(H)=\bigcup_{n=1}^{\infty}\left(0:_{H} \mathcal{R}(\mathfrak{a})_{+}^{n}\right)
$$

Received by the editors May 10, 2002.

2000 Mathematics Subject Classification. Primary 13A02, 13A15, 13E05; Secondary 13A30, $13 \mathrm{D} 45$.

Key words and phrases. Commutative Noetherian ring, associated prime ideal, standard positively graded commutative Noetherian ring, Rees ring, Rees module, associated graded module, Castelnuovo regularity.

The author was partially supported by the Swiss National Foundation (Project numbers 2052762.97 and 2000-042 129.94/1). 
of $H$. (Interpret $a_{\mathcal{R}(\mathfrak{a})_{+}}^{0}(H)$ as $-\infty$ if $\Gamma_{\mathcal{R}(\mathfrak{a})_{+}}(H)=0$. If $H$ is finitely generated, then $a_{\mathcal{R}(\mathfrak{a})_{+}}^{0}(H)$ is an integer or $-\infty$.) We denote by $\operatorname{Var}(\mathfrak{a})$ the variety $\operatorname{Supp}_{A}(A / \mathfrak{a})$ of $\mathfrak{a}$. The following results of McAdam and Eakin are fundamental.

0.1. Theorem (McAdam and Eakin). We have

(i) $\operatorname{Ass}^{*}(\mathfrak{a})=\operatorname{Bss}^{*}(\mathfrak{a}) \cup(\operatorname{Var}(\mathfrak{a}) \cap \operatorname{Ass} A)$ ( 4, Proposition 10 and Corollary 13] );

(ii) $\left(\operatorname{Ass}\left(\mathfrak{a}^{n} / \mathfrak{a}^{n+1}\right)\right)_{n \in \mathbb{N}_{0}}$ is increasing for $n>a_{\mathcal{R}(\mathfrak{a})_{+}}^{0}(\mathcal{G}(\mathfrak{a}))$ (see 4, pp. 71$72])$;

(iii) $\operatorname{Bss}^{*}(\mathfrak{a})=\left\{\mathfrak{P} \cap A: \mathfrak{P} \in \operatorname{Ass}_{\mathcal{R}(\mathfrak{a})}(\mathcal{G}(\mathfrak{a})) \cap \operatorname{Proj}(\mathcal{R}(\mathfrak{a}))\right\}$ (see [4, Proposition 16]).

(In connection with Theorem0.1(ii), it should be noted that if the $i$-th component of the graded module $\left(0: \mathcal{G}(\mathfrak{a}) \mathcal{R}(\mathfrak{a})_{+}\right)$is zero for all $i$ greater than an integer $b$, then $a_{\mathcal{R}(\mathfrak{a})_{+}}^{0}(\mathcal{G}(\mathfrak{a})) \leq b$. This can be established by an easy inductive argument.)

This paper is concerned with the following question: given a prime ideal $\mathfrak{p}$ of $A$ which is known to be in $\operatorname{Ass}^{*}(\mathfrak{a})$, can one identify an integer $n_{\mathfrak{p}}$ with the property that $\mathfrak{p} \in \operatorname{Ass}\left(A / \mathfrak{a}^{n}\right)$ for all $n>n_{\mathfrak{p}}$ ? In other words, can one identify a term of the sequence $\left(\operatorname{Ass}\left(A / \mathfrak{a}^{n}\right)\right)_{n \in \mathbb{N}}$ beyond which $\mathfrak{p}$ will subsequently be an 'ever-present'? The main results of this paper in this direction are as follows. Let $\operatorname{reg}(\mathcal{G}(\mathfrak{a}))$ denote the Castelnuovo regularity of $\mathcal{G}(\mathfrak{a})$.

It is shown in $\$ 2$ that $\mathfrak{p} \in \operatorname{Ass}\left(A / \mathfrak{a}^{n}\right)$ for all $n>\operatorname{reg}(\mathcal{G}(\mathfrak{a}))+1$ if

- $\mathfrak{p}=\mathfrak{P} \cap A$ where $\mathfrak{P} \in \operatorname{Ass}_{\mathcal{R}(\mathfrak{a})}(\mathcal{G}(\mathfrak{a}))$ and ht $\left(\left(\mathfrak{P}+\mathcal{R}(\mathfrak{a})_{+}\right) / \mathfrak{P}\right)=1$,

or

- $\mathfrak{p}$ is a minimal member of the set $\left\{\mathfrak{Q} \cap A: \mathfrak{Q} \in \operatorname{Ass}_{\mathcal{R}(\mathfrak{a})}(\mathcal{G}(\mathfrak{a})) \cap \operatorname{Proj}(\mathcal{R}(\mathfrak{a}))\right\}$,

or

- $\mathfrak{p} \in \operatorname{Ass}^{*}(\mathfrak{a}) \backslash \operatorname{Bss}^{*}(\mathfrak{a})$.

These results are essentially consequences of results in $\$ 1$ about the convergence of the sequence $\left(\operatorname{Ass}_{R_{0}} M_{n}\right)_{n}$, where $M=\bigoplus_{n \in \mathbb{Z}} M_{n}$ is a finitely generated graded module over a positively graded commutative Noetherian ring $R=\bigoplus_{n \in \mathbb{N}_{0}} R_{n}$ which is standard (that is, such that $R=R_{0}\left[R_{1}\right]$ ). These results are perhaps of independent interest. Applications to the convergence of the sequences $\left(\operatorname{Ass}\left(A / \mathfrak{a}^{n}\right)\right)_{n \in \mathbb{N}}$ and $\left(\operatorname{Ass}\left(\mathfrak{a}^{n} / \mathfrak{a}^{n+1}\right)\right)_{n \in \mathbb{N}_{0}}$ are presented in 42 ,

\section{GRAded MOdUles OVER A STANDARD POSITIVELY GRADED RING}

1.1. Notation. Throughout the paper, $R=\bigoplus_{n \in \mathbb{N}_{0}} R_{n}$ will denote a positively graded commutative Noetherian ring which is standard in the sense that $R=$ $R_{0}\left[R_{1}\right]$. We denote the set of graded prime ideals of $R$ by ${ }^{*} \operatorname{Spec}(R)$, and, for a graded ideal $\mathfrak{b}$ of $R$, we will use $* \operatorname{Var}(\mathfrak{b})$ as an abbreviation for $\operatorname{Var}(\mathfrak{b}) \cap * \operatorname{Spec}(R)$. Also, $M=\bigoplus_{n \in \mathbb{Z}} M_{n}$ will denote a graded $R$-module; $M$ will only be assumed to be finitely generated when this is explicitly stated.

We use, for $j \in \mathbb{Z}$, the notation $L_{j}$ to denote the $j$-th component of a $\mathbb{Z}$-graded module $L$, and $(\cdot)(j)$ to denote the $j$-th shift functor on the category of graded $R$-modules and homogeneous homomorphisms (by 'homogeneous' here, we mean 'homogeneous of degree zero'). It will be convenient to have available the concepts of the end and beginning $(\operatorname{beg}(L))$ of the graded $R$-module $L=\bigoplus_{n \in \mathbb{Z}} L_{n}$, which 
are defined by

$$
\operatorname{end}(L):=\sup \left\{n \in \mathbb{Z}: L_{n} \neq 0\right\} \quad \text { and } \quad \operatorname{beg}(L):=\inf \left\{n \in \mathbb{Z}: L_{n} \neq 0\right\} .
$$

(Note that end $(L)$ could be $\infty$, and that the supremum of the empty set of integers is to be taken as $-\infty$; similar comments apply to $\operatorname{beg}(L)$.) For $i \in \mathbb{N}_{0}$, we denote $\operatorname{end}\left(H_{R_{+}}^{i}(M)\right)$ by $a_{R_{+}}^{i}(M)$; when $M$ is finitely generated, this is an integer or $-\infty$.

\subsection{Lemma. We have}

$$
\begin{aligned}
& a_{R_{+}}^{0}(M)=\sup \left\{n \in \mathbb{Z}: \text { there exists } m_{n} \in M_{n}\right. \\
& \left.\qquad \text { with }\left(0:_{R} m_{n}\right) \in \operatorname{Ass}_{R} M \cap{ }^{*} \operatorname{Var}\left(R_{+}\right)\right\} .
\end{aligned}
$$

Proof. If $\mathfrak{p} \in \operatorname{Ass}_{R} M \cap^{*} \operatorname{Var}\left(R_{+}\right)$and $n \in \mathbb{Z}, m_{n} \in M_{n}$ are such that $\left(0:_{R} m_{n}\right)=\mathfrak{p}$, then $0 \neq m_{n} \in \Gamma_{R_{+}}(M)$, and so $n \leq a_{R_{+}}^{0}(M)$. On the other hand, for any integer $b \leq a_{R_{+}}^{0}(M)$, the submodule $N:=\bigoplus_{j>b} \Gamma_{R_{+}}(M)_{j}$ is a non-zero graded $R$-module and so must have an associated prime $\mathfrak{q}$; in fact, $\mathfrak{q}=\left(0: g_{j}\right)$ for some homogeneous element $g_{j}$ of $N$ of degree $j$, say. Since $g_{j}$ is annihilated by some power of $R_{+}$, we must have $\mathfrak{q} \in \operatorname{Ass}_{R} M \cap * \operatorname{Var}\left(R_{+}\right)$.

1.3. Lemma. Let $\mathfrak{p} \in \operatorname{Ass}_{R} M \cap \operatorname{Proj}(R)$. Then there exists $w_{\mathfrak{p}} \in \mathbb{Z}$ such that $\mathfrak{p} \cap R_{0} \in \operatorname{Ass}_{R_{0}} M_{n}$ for all $n \geq w_{\mathfrak{p}}$.

Proof. There exists a homogeneous element $m_{w_{\mathfrak{p}}}$, of degree $w_{\mathfrak{p}}$ say, such that $\left(0:_{R} m_{w_{\mathfrak{p}}}\right)=\mathfrak{p}$. Since $\mathfrak{p} \in \operatorname{Proj}(R)$ and $R$ is standard, there exists $a_{1} \in R_{1} \backslash \mathfrak{p}$, and $\left(0: R a_{1}^{k} m_{w_{\mathfrak{p}}}\right)=\mathfrak{p}$ for all $k \in \mathbb{N}_{0}$. Therefore $\mathfrak{p} \cap R_{0} \in \operatorname{Ass}_{R_{0}} M_{k+w_{\mathfrak{p}}}$ for all $k \in \mathbb{N}_{0}$.

The statement of the next theorem involves the 0-th level anchor points for $M$; see $[5, \S 0]$.

1.4. Theorem. Let $\mathfrak{p}_{0} \in \operatorname{Ass}_{R_{0}} M_{n}$, where $n \in \mathbb{Z}$. Then

(i) $\mathfrak{p}_{0}=\mathfrak{p} \cap R_{0}$ for some $\mathfrak{p} \in \operatorname{Ass}_{R} M \cap \operatorname{Proj}(R)$, and $\mathfrak{p}_{0} \in \operatorname{Ass}_{R_{0}} M_{i}$ for all $i \geq n$;

or

(ii) $\mathfrak{p}_{0}=\mathfrak{p} \cap R_{0}$ for some $\mathfrak{p} \in \operatorname{Ass}_{R} M \cap{ }^{*} \operatorname{Var}\left(R_{+}\right)$, there exists a 0 -th level anchor point $h$ of $\mathfrak{p}$ for $M$ with $n \leq h \leq a^{0}:=a_{R_{+}}^{0}(M)$, and $\mathfrak{p}_{0} \in \operatorname{Ass}_{R_{0}} M_{i}$ for all $i=n, n+1, \ldots, h$.

Note. It should be noted that the conclusions in (i) and (ii) of the above statement could occur simultaneously.

Proof. One can use homogeneous localization at $\mathfrak{p}_{0}+R_{+}$to see that it is enough to prove the theorem under the additional hypotheses that $R$ is *local with unique *maximal ideal $\mathfrak{m}$, and $\mathfrak{m}_{0}:=\mathfrak{m} \cap R_{0}=\mathfrak{p}_{0}$.

There exists $g_{n} \in M_{n}$ such that $\left(0:_{R_{0}} g_{n}\right)=\mathfrak{m}_{0}$. The graded $R$-submodule $R g_{n}$ of $M$ must have an associated prime: thus there exists $k \in \mathbb{N}_{0}$ and a homogeneous element $r_{k} \in R_{k}$ such that $\left(0:_{R} r_{k} g_{n}\right)=\mathfrak{p}$, a graded prime ideal of $R$ with $\mathfrak{p} \cap R_{0}=\mathfrak{m}_{0}$.

First consider the case where $\mathfrak{p} \in \operatorname{Proj}(R)$. Let $j \in \mathbb{N}$. Then, since $R$ is standard, there exists $a_{1} \in R_{1} \backslash \mathfrak{p}$, and $\mathfrak{m}_{0} \subseteq\left(0:_{R_{0}} a_{1}^{j} g_{n}\right)$ and $a_{1}^{j} g_{n} \neq 0$ because $a_{1}^{j} r_{k} g_{n} \neq 0$. Hence $\mathfrak{m}_{0}=\left(0:_{R_{0}} a_{1}^{j} g_{n}\right)$, so that $\mathfrak{m}_{0} \in \operatorname{Ass}_{R_{0}} M_{n+j}$. This is true for all $j \in \mathbb{N}$. 
Now consider the remaining case, where $\mathfrak{p} \in{ }^{*} \operatorname{Var}\left(R_{+}\right)$. We use ${ }^{*} E_{R}(N)$ to denote the *injective envelope of a graded $R$-module $N$. Set $h:=k+n$. In this case, ${ }^{*} E_{R}\left(R r_{k} g_{n}\right)$ is a direct summand of ${ }^{*} E_{R}(M)$, and so the latter graded $R$ module has a direct summand homogeneously isomorphic to ${ }^{*} E_{R}(R / \mathfrak{p})(-h)$. Thus $h$ is a 0 -th level anchor point of $\mathfrak{p}$ for $M$.

Also, it follows from the fact that $R$ is standard that, for each $j=1, \ldots k$, there exists a homogeneous element $r_{j} \in R_{j}$ such that $r_{j} g_{n} \neq 0$. We must therefore have $\mathfrak{m}_{0}=\left(0: R_{0} r_{j} g_{n}\right)$; note also that $r_{j} g_{n} \in M_{n+j}$. Hence $\mathfrak{m}_{0} \in \operatorname{Ass}_{R_{0}} M_{i}$ for all $i=n, n+1, \ldots, n+k=h$. This completes the proof.

In the special case when $M$ is finitely generated, the fact that $\operatorname{Ass}_{R_{0}} M_{n} \subseteq$ $\operatorname{Ass}_{R_{0}} M_{n+1}$ for all $n>a_{R_{+}}^{0}(M)$ is (essentially) proved in 44 pp. 71-72]. The following corollary presents, among other things, the corresponding statement in the general case.

1.5. Corollary. Let the situation be as in Theorem 1.4, and let $n$ be an integer.

(i) $\operatorname{Ass}_{R_{0}} M_{n} \subseteq \operatorname{Ass}_{R_{0}} M_{n+1}$ provided $n$ is not a 0-th level anchor point for M. In particular, $\operatorname{Ass}_{R_{0}} M_{n} \subseteq \operatorname{Ass}_{R_{0}} M_{n+1}$ if $n<\inf \{h: h$ is a 0 -th level anchor point for $M\}$ or if $n>a_{R_{+}}^{0}(M)$, and $\operatorname{Ass}_{R_{0}} M_{n} \subseteq \operatorname{Ass}_{R_{0}} M_{n+1}$ for every value of $n$ if $\Gamma_{R_{+}}(M)=0$.

(ii) We always have $\operatorname{Ass}_{R_{0}} M_{n} \subseteq\left\{\mathfrak{p} \cap R_{0}: \mathfrak{p} \in \operatorname{Ass}_{R} M\right\}$, while

$\operatorname{Ass}_{R_{0}} M_{n} \subseteq\left\{\mathfrak{p} \cap R_{0}: \mathfrak{p} \in \operatorname{Proj}(R) \cap \operatorname{Ass}_{R} M\right\} \quad$ if $n>a_{R_{+}}^{0}(M)$.

(iii) If $\operatorname{Proj}(R) \cap \operatorname{Ass}_{R} M$ is finite (this is the case if $M$ is finitely generated, of course), then

$$
\operatorname{Ass}_{R_{0}} M_{n}=\left\{\mathfrak{p} \cap R_{0}: \mathfrak{p} \in \operatorname{Proj}(R) \cap \operatorname{Ass}_{R} M\right\} \quad \text { for all } n>>0 .
$$

Proof. (i) This all follows from Theorem 1.4 once it has been recalled from [5] Theorem 2.4] that $a_{R_{+}}^{0}(M)$ is the least upper bound of all the 0-th level anchor points for $M$.

(ii) The first claim follows from Theorem 1.4. Furthermore, if $\mathfrak{p}_{0} \in \operatorname{Ass}_{R_{0}} M_{n}$, where $n>a_{R_{+}}^{0}(M)$, then it follows from that theorem that $\mathfrak{p}_{0}=\mathfrak{p} \cap R_{0}$ for some $\mathfrak{p} \in \operatorname{Ass}_{R} M \cap \operatorname{Proj}(R)$, because the conclusion of Theorem 1.4(ii) cannot hold.

(iii) By part (ii), $\operatorname{Ass}_{R_{0}} M_{n} \subseteq\left\{\mathfrak{p} \cap R_{0}: \mathfrak{p} \in \operatorname{Proj}(R) \cap \operatorname{Ass}_{R} M\right\}$ for all $n>$ $a_{R_{+}}^{0}(M)$. Now consider $\mathfrak{p} \in \operatorname{Proj}(R) \cap \operatorname{Ass}_{R} M$. Then $\mathfrak{p} \cap R_{0} \in \operatorname{Ass}_{R_{0}} M_{n}$ for all $n>>$, by Lemma 1.3 Since $\operatorname{Proj}(R) \cap \operatorname{Ass}_{R} M$ is finite, this is enough to establish the claim.

1.6. Remark. In the special case of Corollary 1.5 in which $M$ is finitely generated, there are only finitely many 0 -th level anchor points for $M$, and so there are at most finitely many integers $n$ for which $\operatorname{Ass}_{R_{0}} M_{n} \not \subseteq \operatorname{Ass}_{R_{0}} M_{n+1}$.

The example in which $R=K[X]$, the ring of polynomials over a field $K$, graded in the usual way, and

$$
M=\left(K[X] /\left(X^{t}\right)\right)(t+1) \oplus K[X],
$$

where $t \in \mathbb{N}$, illustrates both possibilities described in the conclusion of Theorem 1.4. Note that -2 is a 0 -th level anchor point of $(X)$ for $M$. Also, the sequence

$$
\left(\operatorname{Ass}_{R_{0}} M_{n} \cap\left\{\mathfrak{p} \cap R_{0}: \mathfrak{p} \in \operatorname{Proj}(R) \cap \operatorname{Ass}_{R} M\right\}\right)_{n=\operatorname{beg}(M), \operatorname{beg}(M)+1, \ldots}
$$

is not increasing in this example. 
The next result uses the ideal transform $D_{R_{+}}(M)$ of $M$ with respect to $R_{+}$; see [2, $§ 2.2$ and 12.4.2].

1.7. Theorem. Let the situation be as in Theorem 1.4. Then

(i) $\operatorname{Ass}_{R} D_{R_{+}}(M)=\operatorname{Proj}(R) \cap \operatorname{Ass}_{R} M$;

(ii) $\operatorname{Ass}_{R_{0}}\left(D_{R_{+}}(M)_{n}\right) \subseteq \operatorname{Ass}_{R_{0}}\left(D_{R_{+}}(M)_{n+1}\right)$ for all $n \in \mathbb{Z}$;

(iii) if $M$ is finitely generated,

$$
\operatorname{Ass}_{R_{0}}\left(D_{R_{+}}(M)_{n}\right)=\left\{\mathfrak{p} \cap R_{0}: \mathfrak{p} \in \operatorname{Proj}(R) \cap \operatorname{Ass}_{R} M\right\} \quad \text { for all } n>>0 .
$$

Proof. Set $\bar{M}:=M / \Gamma_{R_{+}}(M)$, and note that, by [2, 2.1.12],

$$
\operatorname{Ass}_{R}(\bar{M})=\operatorname{Proj}(R) \cap \operatorname{Ass}_{R} M .
$$

The exact sequence

$$
0 \longrightarrow \bar{M} \longrightarrow D_{R_{+}}(M) \longrightarrow H_{R_{+}}^{1}(M) \longrightarrow 0
$$

of graded $R$-modules and homogeneous homomorphisms, together with the fact ([2] 2.2.8(iv)]) that $\Gamma_{R_{+}}\left(D_{R_{+}}(M)\right)=0$, now shows that $\operatorname{Ass}_{R} D_{R_{+}}(M)=\operatorname{Ass}_{R} \bar{M}=$ $\operatorname{Proj}(R) \cap \operatorname{Ass}_{R} M$. Note that each associated prime ideal of the $R$-module $H_{R_{+}}^{1}(M)$ must contain $R_{+}$. This proves part (i).

Since $a_{R_{+}}^{0}\left(D_{R_{+}}(M)\right)=\operatorname{end}\left(H_{R_{+}}^{0}\left(D_{R_{+}}(M)\right)\right)=-\infty$, the claim in part (ii) follows from Corollary 1.5(i), while part (iii) follows from part (i) and Corollary 1.5(iii).

For the remainder of this section, we shall suppose that the graded $R$-module $M$ is finitely generated. In the situation of Corollary $[1.5$ it would be interesting to have some estimate of the point of the sequence $\left(\operatorname{Ass}_{R_{0}} M_{n}\right)_{n=\operatorname{beg}(M), \operatorname{beg}(M)+1, \ldots}$ at which the ultimate constant value is first attained. A related, but perhaps easier, question is the following: given $\mathfrak{p} \in \operatorname{Proj}(R) \cap \operatorname{Ass}_{R} M$, so that, by Corollary 1.5 (iii), $\mathfrak{p}_{0}:=\mathfrak{p} \cap R_{0}$ belongs to all except finitely many terms of the sequence, can one estimate an $n_{0} \in \mathbb{Z}$ such that $\mathfrak{p}_{0} \in \operatorname{Ass}_{R_{0}} M_{n}$ for all $n>n_{0}$ ? We next present two results of this type. For the first, recall the definition of the Castelnuovo regularity $\operatorname{reg}(M)$ of $M$.

It is well known that $a_{R_{+}}^{i}(M):=$ end $\left(H_{R_{+}}^{i}(M)\right)<\infty$ for all $i \in \mathbb{N}_{0}$. Also, $H_{R_{+}}^{i}(M)=0$ for $i>\operatorname{ara}\left(R_{+}\right)$, the arithmetic rank of $R_{+}$; see [2, §3.3]. The Castelnuovo regularity $\operatorname{reg}(M)$ of $M$ can be defined by

$$
\operatorname{reg}(M):=\sup \left\{a_{R_{+}}^{i}(M)+i: i \in \mathbb{N}_{0}\right\}
$$

this is either an integer or $-\infty$. (See [2, Chapter 15].) Note that $a_{R_{+}}^{0}(M) \leq \operatorname{reg}(M)$.

1.8. Proposition. Assume that the graded $R$-module $M=\bigoplus_{n \in \mathbb{Z}} M_{n}$ is finitely generated. Suppose that $\mathfrak{p}_{0}$ is a minimal member of the set

$$
\left\{\mathfrak{q} \cap R_{0}: \mathfrak{q} \in \operatorname{Proj}(R) \cap \operatorname{Ass}_{R} M\right\} .
$$

Then $\mathfrak{p}_{0} \in \operatorname{Ass}_{R_{0}} M_{n}$ for all $n>\operatorname{reg}(M)$.

Proof. One can use homogeneous localization at $\mathfrak{p}_{0} R+R_{+}$to see that it is enough to prove the lemma under the additional hypothesis that $R$ is *local with unique *maximal ideal $\mathfrak{m}$, and that $\mathfrak{m}_{0}:=\mathfrak{m} \cap R_{0}=\mathfrak{p}_{0}$.

Suppose that there exists $i \in \mathbb{N}$ such that $\mathfrak{m}_{0} \notin \operatorname{Ass}_{R_{0}} M_{\operatorname{reg}(M)+i}$. Then, by Corollary 1.5, we have

$$
\operatorname{Ass}_{R_{0}} M_{\mathrm{reg}(M)+i}=\emptyset
$$


so that $M_{\mathrm{reg}(M)+i}=0$. But $M$ can be generated by homogeneous elements of degrees not exceeding $\operatorname{reg}(M)$ (by [2, 15.3.1]). Since $R$ is homogeneous, it follows from this that, since $M_{\mathrm{reg}}(M)+i=0$, we must have $M_{\mathrm{reg}}(M)+n=0$ for every $n \geq i$. However, Corollary 1.5 shows that $\operatorname{Ass}_{R_{0}} M_{\operatorname{reg}(M)+n}=\left\{\mathfrak{m}_{0}\right\}$ for all $n>>0$, and so we have arrived at a contradiction.

1.9. Proposition. Assume that the graded $R$-module $M=\bigoplus_{n \in \mathbb{Z}} M_{n}$ is finitely generated.

Let $\mathfrak{p} \in \operatorname{Proj}(R) \cap \operatorname{Ass}_{R} M ;$ set $\mathfrak{p}_{0}=\mathfrak{p} \cap R_{0}$ and suppose that

$$
\operatorname{ht}\left(\left(\mathfrak{p}+R_{+}\right) / \mathfrak{p}\right)=1 \text {. }
$$

Then $\mathfrak{p}_{0} \in \operatorname{Ass}_{R_{0}} M_{n}$ for all $n>\max \left\{a_{R_{+}}^{0}(M), a_{R_{+}}^{1}(M)\right\}$.

Proof. Again, one can use homogeneous localization at $\mathfrak{p}_{0} R+R_{+}$to see that it is enough to prove the lemma under the additional hypothesis that $R$ is *local with unique ${ }^{*}$ maximal ideal $\mathfrak{m}$, and that $\mathfrak{m}_{0}:=\mathfrak{m} \cap R_{0}=\mathfrak{p} \cap R_{0}$.

Set $b:=\sup \left\{a_{R_{+}}^{0}(M), a_{R_{+}}^{1}(M)\right\}$ and $\bar{M}:=M / \Gamma_{R_{+}}(M)$. Let $\pi: M \longrightarrow \bar{M}$ denote the natural epimorphism. Since $\bar{M}$ is $R_{+}$-torsion-free, it follows (from 3 . 1.5.10], for example) that there exists $d \in \mathbb{N}$ and $r_{d} \in R_{d} \backslash \mathfrak{p}$ such that $r_{d}$ is a non-zerodivisor on $\bar{M}$.

Since end $\left(\Gamma_{R_{+}}(M)\right) \leq b$, it follows from an elementary calculation that both the kernel and the cokernel of the homogeneous homomorphism

$$
{ }^{*} \operatorname{Hom}_{R}(R / \mathfrak{p}, \pi):{ }^{*} \operatorname{Hom}_{R}(R / \mathfrak{p}, M) \longrightarrow{ }^{*} \operatorname{Hom}_{R}(R / \mathfrak{p}, \bar{M})
$$

have ends not exceeding $b$. We wish to show that the kernel and the cokernel of the homogeneous homomorphism $* \operatorname{Hom}_{R}(R / \mathfrak{p}, M) \stackrel{r_{d}}{\longrightarrow} * \operatorname{Hom}_{R}(R / \mathfrak{p}, M)(d)$ both have ends not exceeding $b$. It is therefore sufficient, in order to achieve this, to show that the kernel $K$ and the cokernel $C$ of the homogeneous homomorphism * $\operatorname{Hom}_{R}(R / \mathfrak{p}, \bar{M}) \stackrel{r_{d}}{\longrightarrow} * \operatorname{Hom}_{R}(R / \mathfrak{p}, \bar{M})(d)$ both have ends not exceeding $b$. In this case, $K=0$, because $r_{d}$ is a non-zerodivisor on $\bar{M}$.

The exact sequence

$$
0 \longrightarrow \bar{M} \stackrel{r_{d}}{\longrightarrow} \bar{M}(d) \longrightarrow\left(\bar{M} / r_{d} \bar{M}\right)(d) \longrightarrow 0
$$

induces an exact sequence of graded $R$-modules and homogeneous homomorphisms

$$
0 \rightarrow{ }^{*} \operatorname{Hom}_{R}(R / \mathfrak{p}, \bar{M}) \stackrel{r_{d}}{\longrightarrow} * \operatorname{Hom}_{R}(R / \mathfrak{p}, \bar{M})(d) \rightarrow{ }^{*} \operatorname{Hom}_{R}\left(R / \mathfrak{p}, \bar{M} / r_{d} \bar{M}\right)(d) .
$$

Hence end $(C) \leq \operatorname{end}\left({ }^{*} \operatorname{Hom}_{R}\left(R / \mathfrak{p}, \bar{M} / r_{d} \bar{M}\right)(d)\right) \leq$ end $\left(\Gamma_{\mathfrak{p}}\left(\bar{M} / r_{d} \bar{M}\right)(d)\right)$. Now

$$
\Gamma_{\mathfrak{p}}\left(\bar{M} / r_{d} \bar{M}\right)=\Gamma_{\mathfrak{p}+R r_{d}}\left(\bar{M} / r_{d} \bar{M}\right)
$$

But $\sqrt{\mathfrak{p}+R r_{d}}=\mathfrak{m}$, in view of the hypotheses about $\mathfrak{p}$. Hence

$$
\operatorname{end}(C) \leq \text { end }\left(\Gamma_{R_{+}}\left(\bar{M} / r_{d} \bar{M}\right)(d)\right) \text {. }
$$

Use again the exact sequence $0 \longrightarrow \bar{M} \stackrel{r_{d}}{\longrightarrow} \bar{M}(d) \longrightarrow\left(\bar{M} / r_{d} \bar{M}\right)(d) \longrightarrow 0$, this time in conjunction with the fact that $\Gamma_{R_{+}}(\bar{M})=0$, to see that $\Gamma_{R_{+}}\left(\bar{M} / r_{d} \bar{M}\right)(d)$ is homogeneously isomorphic to a graded submodule of $H_{R_{+}}^{1}(\bar{M})$. Since the latter graded module is homogeneously isomorphic to $H_{R_{+}}^{1}(M)$, it follows that end $(C) \leq$ $b$.

It therefore follows that

$$
{ }^{*} \operatorname{Hom}_{R}(R / \mathfrak{p}, M)_{n+d}=r_{d} * \operatorname{Hom}_{R}(R / \mathfrak{p}, M)_{n} \quad \text { for all } n>b .
$$


Since $\mathfrak{p} \in \operatorname{Ass}_{R} M$, there exists a homogeneous element $g_{j}$ of $M_{j}$ (for some $j \in \mathbb{Z}$ ) with annihilator exactly $\mathfrak{p}$; since $R$ is standard, there exists $r_{1}^{\prime} \in R_{1} \backslash \mathfrak{p}$. Multiplication of $g_{j}$ by $r_{1}^{\prime}$ produces another homogeneous element, of degree $j+1$, also with annihilator exactly $\mathfrak{p}$. The last display therefore shows that there is an element of $M_{b+1}$ with annihilator exactly $\mathfrak{p}$, and the claim follows easily from these observations.

\section{Applichtion to Asymptotic PRimes}

In this section, we apply the results of $\$ 1$ to obtain some results about the convergence of the sequences $\left(\operatorname{Ass}\left(N / \mathfrak{a}^{n} N\right)\right)_{n \in \mathbb{N}}$ and $\left(\operatorname{Ass}\left(\mathfrak{a}^{n} N / \mathfrak{a}^{n+1} N\right)\right)_{n \in \mathbb{N}_{0}}$, where $N$ is a finitely generated module over the commutative Noetherian $\operatorname{ring} A$ and $\mathfrak{a}$ is an ideal of $A$. (This notation will be in force for this entire section.) Denote the ultimate constant values of these sequences by $\operatorname{Ass}^{*}(\mathfrak{a}, N)$ and $\operatorname{Bss}^{*}(\mathfrak{a}, N)$, respectively. Denote by $\mathcal{G}(\mathfrak{a}, N)$ the associated graded module of $\mathfrak{a}$ with respect to $N$. For each $t \in \mathbb{N}$, let $\mathcal{G}(\mathfrak{a}, N, t)$ denote the graded $\mathcal{R}(\mathfrak{a})$-module

$$
\cdots \oplus 0 \oplus 0 \oplus N / \mathfrak{a} N \oplus N / \mathfrak{a}^{2} N \oplus \cdots \oplus N / \mathfrak{a}^{t} N \oplus \mathfrak{a} N / \mathfrak{a}^{t+1} N \oplus \mathfrak{a}^{2} N / \mathfrak{a}^{t+2} N \oplus \cdots,
$$

where it is to be understood that $N / \mathfrak{a} N$ is the component of degree 1 . (Thus $\mathcal{G}(\mathfrak{a}, N, 1)=\mathcal{G}(\mathfrak{a}, N)(-1)$.)

Also, let $N[X]$ denote the $A[X]$-module $N \otimes_{A} A[X]$ (where $X$ is an indeterminate over $A$ ); in $N[X]$, denote $y \otimes X^{j}$, where $y \in N$ and $j \in \mathbb{N}_{0}$, by $y X^{j}$. Note that $N[X]$ can be regarded as an $\mathcal{R}(\mathfrak{a})$-module by restriction of scalars. Let

$$
\mathcal{R}(\mathfrak{a}, N)=\left\{\sum_{j=0}^{t} n_{j} X^{j}: t \in \mathbb{N}_{0}, n_{j} \in \mathfrak{a}^{j} N(0 \leq j \leq t)\right\},
$$

an $\mathcal{R}(\mathfrak{a})$-submodule of $N[X]$ which we refer to as the Rees module of $\mathfrak{a}$ with respect to $N$.

We begin by applying results from $\$ 1$ to the graded $\mathcal{R}(\mathfrak{a})$-modules $\mathcal{G}(\mathfrak{a}, N)$ and $N[X] / \mathcal{R}(\mathfrak{a}, N)$ to obtain results about the convergence of the sequences

$$
\left(\operatorname{Ass}\left(\mathfrak{a}^{n} N / \mathfrak{a}^{n+1} N\right)\right)_{n \in \mathbb{N}_{0}} \text { and }\left(\operatorname{Ass}\left(N / \mathfrak{a}^{n} N\right)\right)_{n \in \mathbb{N}} .
$$

The first two results are such consequences of Lemma 1.3.

2.1. Proposition. Let $\mathfrak{P} \in \operatorname{Ass}_{\mathcal{R}(\mathfrak{a})}(\mathcal{G}(\mathfrak{a}, N)) \cap \operatorname{Proj}(\mathcal{R}(\mathfrak{a}))$. Then there exists $w_{\mathfrak{P}} \in \mathbb{N}_{0}$ such that

$$
\mathfrak{P} \cap A \in \operatorname{Ass}\left(\mathfrak{a}^{n} N / \mathfrak{a}^{n+1} N\right) \text { for all } n \geq w_{\mathfrak{P}} .
$$

2.2. Proposition. Let $\mathfrak{P} \in \operatorname{Ass}_{\mathcal{R}(\mathfrak{a})}(N[X] / \mathcal{R}(\mathfrak{a}, N)) \cap \operatorname{Proj}(\mathcal{R}(\mathfrak{a}))$. Then there exists $u_{\mathfrak{P}} \in \mathbb{N}$ such that $\mathfrak{P} \cap A \in \operatorname{Ass}\left(N / \mathfrak{a}^{n} N\right)$ for all $n \geq u_{\mathfrak{P}}$.

The next result is an immediate consequence of Theorem 1.4 .

2.3. Theorem. Let $\mathfrak{p} \in \operatorname{Ass}\left(\mathfrak{a}^{n} N / \mathfrak{a}^{n+1} N\right)$, where $n \in \mathbb{N}_{0}$. Then

(i) $\mathfrak{p}=\mathfrak{P} \cap A$ for some $\mathfrak{P} \in \operatorname{Ass}_{\mathcal{R}(\mathfrak{a})}(\mathcal{G}(\mathfrak{a}, N)) \cap \operatorname{Proj}(\mathcal{R}(\mathfrak{a}))$ and

$$
\mathfrak{p} \in \operatorname{Ass}\left(\mathfrak{a}^{i} N / \mathfrak{a}^{i+1} N\right) \text { for all } i \geq n ;
$$

or

(ii) $\mathfrak{p}=\mathfrak{P} \cap A$ for some $\mathfrak{P} \in \operatorname{Ass}_{\mathcal{R}(\mathfrak{a})}(\mathcal{G}(\mathfrak{a}, N)) \cap{ }^{*} \operatorname{Var}\left(\mathcal{R}(\mathfrak{a})_{+}\right)$, there exists a 0 -th level anchor point $h$ of $\mathfrak{P}$ for $\mathcal{G}(\mathfrak{a}, N)$ with $n \leq h \leq a^{0}:=$ $a_{\mathcal{R}(\mathfrak{a})_{+}}^{0}(\mathcal{G}(\mathfrak{a}, N))$, and $\mathfrak{p} \in \operatorname{Ass}\left(\mathfrak{a}^{i} N / \mathfrak{a}^{i+1} N\right)$ for all $i=n, n+1, \ldots, h$. 
The next result is an immediate consequence of Corollary 1.5 .

2.4. Proposition. (i) $\operatorname{Ass}\left(\mathfrak{a}^{n} N / \mathfrak{a}^{n+1} N\right) \subseteq \operatorname{Ass}\left(\mathfrak{a}^{n+1} N / \mathfrak{a}^{n+2} N\right)$ for all $n>$ $a_{\mathcal{R}(\mathfrak{a})_{+}}^{0}(\mathcal{G}(\mathfrak{a}, N))$.

(ii) $\operatorname{Ass}\left(\mathfrak{a}^{n} N / \mathfrak{a}^{n+1} N\right)=\left\{\mathfrak{P} \cap A: \mathfrak{P} \in \operatorname{Ass}_{\mathcal{R}(\mathfrak{a})}(\mathcal{G}(\mathfrak{a}, N)) \cap \operatorname{Proj}(\mathcal{R}(\mathfrak{a}))\right\}$ for all $n>>0$.

Given a $\mathfrak{P} \in \operatorname{Ass}_{\mathcal{R}(\mathfrak{a})}(\mathcal{G}(\mathfrak{a}, N)) \cap \operatorname{Proj}(\mathcal{R}(\mathfrak{a}))$, can one identify a term of the sequence

$$
\left(\operatorname{Ass}\left(\mathfrak{a}^{n} N / \mathfrak{a}^{n+1} N\right)\right)_{n \in \mathbb{N}_{0}}
$$

beyond which $\mathfrak{P} \cap A$ will subsequently be an 'ever-present'? The next two results, which follow from Propositions [1.8] and[1.9, give such estimates in special cases.

2.5. Proposition. If $\mathfrak{p}$ is a minimal member of the set

$$
\left\{\mathfrak{Q} \cap A: \mathfrak{Q} \in \operatorname{Ass}_{\mathcal{R}(\mathfrak{a})}(\mathcal{G}(\mathfrak{a}, N)) \cap \operatorname{Proj}(\mathcal{R}(\mathfrak{a}))\right\},
$$

then $\mathfrak{p} \in \operatorname{Ass}\left(\mathfrak{a}^{n} N / \mathfrak{a}^{n+1} N\right)$ for all $n>\operatorname{reg}(\mathcal{G}(\mathfrak{a}, N))$.

2.6. Proposition. Let $\mathfrak{P} \in \operatorname{Ass}_{\mathcal{R}(\mathfrak{a})}(\mathcal{G}(\mathfrak{a}, N)) \cap \operatorname{Proj}(\mathcal{R}(\mathfrak{a}))$ and suppose that ht $\left(\left(\mathfrak{P}+\mathcal{R}(\mathfrak{a})_{+}\right) / \mathfrak{P}\right)=1$. Then $\mathfrak{p}:=\mathfrak{P} \cap A \in \operatorname{Ass}\left(\mathfrak{a}^{n} N / \mathfrak{a}^{n+1} N\right)$ for all $n>$ $\max \left\{a_{\mathcal{R}(\mathfrak{a})_{+}}^{0}(\mathcal{G}(\mathfrak{a}, N)), a_{\mathcal{R}(\mathfrak{a})_{+}}^{1}(\mathcal{G}(\mathfrak{a}, N))\right\}$.

2.7. Lemma. We have $\operatorname{Ass}_{A}\left(N / \mathfrak{a}^{n} N\right) \subseteq\left\{\mathfrak{P} \cap A: \mathfrak{P} \in \operatorname{Ass}_{\mathcal{R}(\mathfrak{a})}(\mathcal{G}(\mathfrak{a}, N))\right\}$ for all $n \in \mathbb{N}$.

Proof. When $t>1$, there is an exact sequence

$$
0 \longrightarrow \mathcal{G}(\mathfrak{a}, N, t-1) \longrightarrow \mathcal{G}(\mathfrak{a}, N, t) \longrightarrow \mathcal{G}(\mathfrak{a}, N)(-t) \longrightarrow 0
$$

of graded $\mathcal{R}(\mathfrak{a})$-modules and homogeneous homomorphisms, and so it follows easily by induction on $t$ that, for all $t \in \mathbb{N}$, the graded $\mathcal{R}(\mathfrak{a})$-module $\mathcal{G}(\mathfrak{a}, N, t)$ is finitely generated and such that

$$
\operatorname{Ass}_{\mathcal{R}(\mathfrak{a})}(\mathcal{G}(\mathfrak{a}, N, t))=\operatorname{Ass}_{\mathcal{R}(\mathfrak{a})}(\mathcal{G}(\mathfrak{a}, N)) .
$$

Since, for each $n \in \mathbb{N}$, the $A$-module $N / \mathfrak{a}^{n} N$ is the $n$-th component of $\mathcal{G}(\mathfrak{a}, N, n)$, the result follows from Corollary 1.5 (ii).

2.8. Proposition. Let $\mathfrak{p} \in \operatorname{Ass}^{*}(\mathfrak{a}, N) \backslash \operatorname{Bss}^{*}(\mathfrak{a}, N)$. Then $\mathfrak{p} \in \operatorname{Ass}\left(N / \mathfrak{a}^{n} N\right)$ for all positive integers $n$ with $n>a_{\mathcal{R}(\mathfrak{a})_{+}}^{0}(\mathcal{G}(\mathfrak{a}, N))$.

Proof. Suppose there exists a positive integer $n$ with $n>a_{\mathcal{R}(\mathfrak{a})_{+}}^{0}(\mathcal{G}(\mathfrak{a}, N))$ such that $\mathfrak{p} \notin \operatorname{Ass}\left(N / \mathfrak{a}^{n} N\right)$. Since $\mathfrak{p} \in \operatorname{Ass}^{*}(\mathfrak{a}, N)$, there exists $t \in \mathbb{N}$ such that $\mathfrak{p} \in$ $\operatorname{Ass}\left(N / \mathfrak{a}^{n+t} N\right)$ but $\mathfrak{p} \notin \operatorname{Ass}\left(N / \mathfrak{a}^{n+t-1} N\right)$. Now we cannot have

$$
\mathfrak{p} \in \operatorname{Ass}\left(\mathfrak{a}^{n+t-1} N / \mathfrak{a}^{n+t} N\right)
$$

since, by Proposition 2.4(i), that would imply that $\mathfrak{p} \in \operatorname{Bss}^{*}(\mathfrak{a}, N)$. It therefore follows from the exact sequence

$$
0 \longrightarrow \mathfrak{a}^{n+t-1} N / \mathfrak{a}^{n+t} N \longrightarrow N / \mathfrak{a}^{n+t} N \longrightarrow N / \mathfrak{a}^{n+t-1} N \longrightarrow 0
$$

that $\mathfrak{p} \in \operatorname{Ass}\left(N / \mathfrak{a}^{n+t-1} N\right)$, a contradiction. Hence $\mathfrak{p} \in \operatorname{Ass}\left(N / \mathfrak{a}^{m} N\right)$ for all positive integers $m$ with $m>a_{\mathcal{R}(\mathfrak{a})_{+}}^{0}(\mathcal{G}(\mathfrak{a}, N))$. 
Actually, one can improve on Proposition 2.8 It is easy to modify the argument of N. V. Trung in [6], Theorem 3.1(i)] to show that $a_{\mathcal{R}(\mathfrak{a})_{+}}^{0}(\mathcal{R}(\mathfrak{a}, N)) \leq$ $a_{\mathcal{R}(\mathfrak{a})_{+}}^{0}(\mathcal{G}(\mathfrak{a}, N))$.

2.9. Proposition. Let $\mathfrak{p} \in \operatorname{Ass}^{*}(\mathfrak{a}, N) \backslash \operatorname{Bss}^{*}(\mathfrak{a}, N)$. Then $\mathfrak{p} \in \operatorname{Ass}\left(N / \mathfrak{a}^{n} N\right)$ for all positive integers $n$ with $n>a_{\mathcal{R}(\mathfrak{a})_{+}}^{0}(\mathcal{R}(\mathfrak{a}, N))$.

Proof. An obvious modification of the arguments in 4, Lemma 11, Lemma 12, Corollary 13] will show that $\operatorname{Ass}^{*}(\mathfrak{a}, N) \backslash \operatorname{Bss}^{*}(\mathfrak{a}, N) \subseteq \operatorname{Ass}_{A} N \cap \operatorname{Var}(\mathfrak{a})$. Let $\mathfrak{p} \in$ $\operatorname{Ass}_{A} N \cap \operatorname{Var}(\mathfrak{a})$, so that there exists $w \in N$ with $\left(0::_{A} w\right)=\mathfrak{p} \supseteq \mathfrak{a}$. Then $\left(0:_{A[X]} w X^{j}\right)=\mathfrak{p} A[X]$ for all $j \in \mathbb{N}_{0}$, and so $\left(0:_{\mathcal{R}(\mathfrak{a})} w X^{j}\right)=\mathfrak{p} A[X] \cap \mathcal{R}(\mathfrak{a})$ for all $j \in \mathbb{N}_{0}$. However, $\mathfrak{p} A[X] \cap \mathcal{R}(\mathfrak{a}) \in{ }^{*} \operatorname{Var}\left(\mathcal{R}(\mathfrak{a})_{+}\right)$because $\mathfrak{a} \subseteq \mathfrak{p}$. Hence, for $j \in \mathbb{N}$ with $j>a_{\mathcal{R}(\mathfrak{a})_{+}}^{0}(\mathcal{R}(\mathfrak{a}, N))$, the $\mathcal{R}(\mathfrak{a})$-submodule $\mathcal{R}(\mathfrak{a}) w X^{j}$ of $N[X]$ must have zero intersection with $\mathcal{R}(\mathfrak{a}, N)$. Consideration of the exact sequence

$$
0 \longrightarrow \mathcal{R}(\mathfrak{a}, N) \longrightarrow N[X] \longrightarrow N[X] / \mathcal{R}(\mathfrak{a}, N) \longrightarrow 0
$$

of graded $\mathcal{R}(\mathfrak{a})$-modules and homogeneous homomorphisms therefore shows that the natural image of $w X^{j}$ in $N[X] / \mathcal{R}(\mathfrak{a}, N)$ has annihilator $\mathfrak{p} A[X] \cap \mathcal{R}(\mathfrak{a})$ for all $j \in \mathbb{N}$ with $j>a_{\mathcal{R}(\mathfrak{a})_{+}}^{0}(\mathcal{R}(\mathfrak{a}, N))$. The claim follows from this.

We end the paper by drawing together some consequences of Propositions 2.5 2.6 and 2.8 in order to list some circumstances in which a $\mathfrak{p} \in \operatorname{Ass}^{*}(\mathfrak{a})$ is an everpresent from the $(r+2)$-th term onwards of the sequence $\left(\operatorname{Ass}\left(A / \mathfrak{a}^{n}\right)\right)_{n \in \mathbb{N}}$, where $r:=\operatorname{reg}(\mathcal{G}(\mathfrak{a}))$.

2.10. Theorem. Let $\mathfrak{p} \in \operatorname{Ass}^{*}(\mathfrak{a})$.

(i) Suppose $\mathfrak{p}=\mathfrak{P} \cap A$ where $\mathfrak{P} \in \operatorname{Ass}_{\mathcal{R}(\mathfrak{a})}(\mathcal{G}(\mathfrak{a})) \cap \operatorname{Proj}(\mathcal{R}(\mathfrak{a})$ ) (see Theorem 0.1 (iii)). Then $\mathfrak{p} \in \operatorname{Ass}\left(A / \mathfrak{a}^{n}\right)$ for all $n>\operatorname{reg}(\mathcal{G}(\mathfrak{a}))+1$ if

- $\mathfrak{p}$ is a minimal member of the set

$$
\left\{\mathfrak{Q} \cap A: \mathfrak{Q} \in \operatorname{Ass}_{\mathcal{R}(\mathfrak{a})}(\mathcal{G}(\mathfrak{a})) \cap \operatorname{Proj}(\mathcal{R}(\mathfrak{a}))\right\},
$$

or

- ht $\left(\left(\mathfrak{P}+\mathcal{R}(\mathfrak{a})_{+}\right) / \mathfrak{P}\right)=1$

(ii) If $\mathfrak{p} \in \operatorname{Ass}^{*}(\mathfrak{a}) \backslash \operatorname{Bss}^{*}(\mathfrak{a})$, then $\mathfrak{p} \in \operatorname{Ass}\left(A / \mathfrak{a}^{n}\right)$ for all $n>\operatorname{reg}(\mathcal{G}(\mathfrak{a}))$.

\section{REFERENCES}

[1] M. Brodmann, Asymptotic stability of $\operatorname{Ass}\left(M / I^{n} M\right)$, Proc. Amer. Math. Soc. 74 (1979), 1618. MR 80c: 13012

[2] M. P. Brodmann and R. Y. Sharp, Local cohomology: an algebraic introduction with geometric applications, Cambridge University Press, 1998. MR 99h:13020

[3] W. Bruns and J. Herzog, Cohen-Macaulay rings, Cambridge University Press, 1993. MR 95h:13020

[4] S. McAdam and P. Eakin, The asymptotic Ass, J. Algebra 61 (1979), 71-81. MR 81f:13001

[5] R. Y. Sharp, Bass numbers in the graded case, a-invariant formulas, and an analogue of Faltings' Annihilator Theorem, J. Algebra 222 (1999), 246-270. MR 2000j:13027

[6] N. V. Trung, The Castelnuovo regularity of the Rees algebra and the associated graded ring, Transactions Amer. Math. Soc. 350 (1998), 2813-2832. MR 98j:13006

Department of Pure Mathematics, University of Sheffield, Hicks Building, Sheffield S3 7RH, United Kingdom

E-mail address: R.Y.Sharp@sheffield.ac.uk 